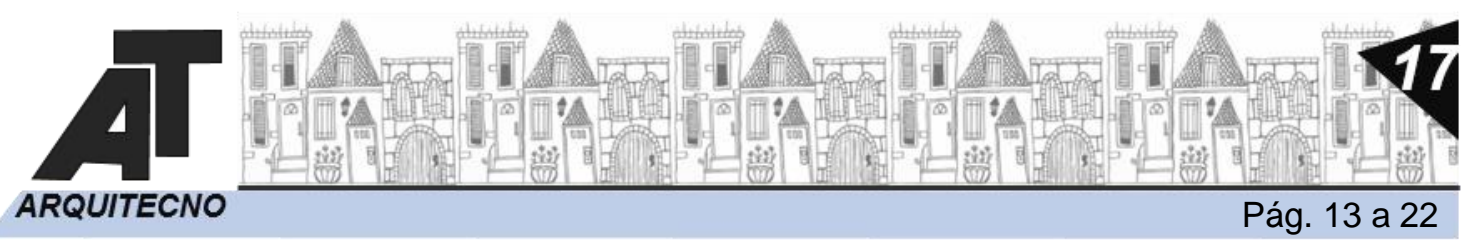

\title{
La cuestión de la infravivienda, un estudio de caso en la ciudad de Barcelona
}

The issue of substandard housing, a case study in the city of Barcelona

\author{
Còssima Cornadón ${ }^{1}$, Pilar Garcia-Almirall ${ }^{1}$, Jordi Casals ${ }^{2}$ y Amaya Caballero ${ }^{1}$ \\ 1Departamento de Tecnología de la Arquitectura, ETSAB, Universidad Politécnica de Cataluña, \\ Barcelona, España \\ 'Laboratorio Virtual de Innovación Modelando la Arquitectura y la Ciudad VIMAC, ETSAB, \\ Universidad Politécnica de Cataluña, Barcelona, España \\ cossima.cornado@upc.edu
}

\section{RESUMEN}

El concepto de infravivienda puede variar notablemente dependiendo del contexto urbano y social de cada lugar, éste se suele referir a aquel tipo de edificación y/o vivienda cuyos niveles de habitabilidad se encuentran por debajo de unos estándares mínimos establecidos, generalmente, en textos normativos. Su aparición a menudo va asociada a procesos de urbanización irregulares y al uso de vivienda de espacios no concebidos para ello, relacionándose, a menudo con situaciones de vulnerabilidad socioresidencial. El caso que aquí se presenta se centra en el barrio del Carmel en Barcelona, caracterizado por un crecimiento urbano en pendiente y la sospecha de la existencia situaciones de infravivienda. Se plantean como objetivos profundizar en el conocimiento de las condiciones de habitabilidad y estado de conservación de las viviendas del barrio para realizar un diagnóstico global de él. Para ello se diseña y emplea una metodología de estudio basada en una combinación de explotación de datos a partir de indicadores y campañas in situ. Los resultados permiten la detección de situaciones de infravivienda, la caracterización de las principales situaciones que la provocan y la categorización y priorización en la necesidad de actuación e intervención por parte de programas públicos de ayuda a la rehabilitación.

\section{ABSTRACT}

The concept of substandard housing can vary notably depending on the urban and social context of each place, it usually refers to that type of building and / or housing whose levels of habitability are below the minimum standards established, generally, in normative texts. Its appearance is often associated with irregular urbanization processes and the use of housing in spaces not designed for it, often being related to situations of socio-residential vulnerability. The case presented here focuses on the Carmel neighborhood in Barcelona, characterized by sloping urban growth and the suspicion of the existence of sub-housing situations. The objectives are to deepen the knowledge of the habitability conditions and state of conservation of the homes in the neighborhood to carry out a global diagnosis of it. To do this, a study methodology based on a combination of data exploitation based on indicators and on-site campaigns is designed and used. The results allow the detection of substandard housing situations, the characterization of the main situations that cause them and the categorization and prioritization of the need for action and intervention by public programs to aid rehabilitation.

PALABRAS CLAVE: diagnosis edificación, edificación residencial, habitabilidad, parque residencial, rehabilitación

KEY WORDS: building diagnosis, residential buildings, habitability, building stock, rehabilitation

FECHA DE RECEPCIÓN: 13/11/2020| FECHA DE ACEPTACIÓN: 12/5/2021

DOI: http://dx.doi.org/10.30972/arq.0174979 


\section{INTRODUCCIÓN}

El concepto de infravivienda puede ser bastante amplio. Por lo general, se entiende como infravivienda aquella que no alcanza unas condiciones mínimas de habitabilidad. En el caso de España, estas condiciones quedan recogidas en el Real Decreto Legislativo 7/2015, de 30 de octubre, por el que se aprueba el texto refundido de la Ley de Suelo y Rehabilitación Urbana (Gobierno de España, 2015) donde se define infravivienda como "edificación, o parte de ella, destinada a vivienda, que no reúne las condiciones mínimas exigidas de conformidad con la legislación aplicable. En todo caso, se entenderá que no reúnen dichas condiciones las viviendas que incumplan los requisitos de superficie, número, dimensión y características de las piezas habitables, las que presenten deficiencias graves en sus dotaciones e instalaciones básicas y las que no cumplan los requisitos mínimos de seguridad, accesibilidad universal y habitabilidad exigibles a la edificación".

Las situaciones de existencia de infravivienda suelen ir asociadas a circunstancias sociales complejas ligadas a poblaciones vulnerables (Aragonés, J. ed. al. 1992; Alcalá, L. ed. al. 2013). En dicho contexto se combinan usos de vivienda en espacios no concebidos para ello, situaciones de hacinamiento y falta de inversión en el mantenimiento de los edificios, dando lugar a situaciones de degradación general de las viviendas.

Desde el consistorio de Barcelona en 2015 se inició un programa de ayudas sociales a los barrios llamado Pla de Barris (Ayuntamiento de Barcelona, 2015). En este contexto se elaboró un mapa de vulnerabilidad socioresidencial de la ciudad de Barcelona (Garcia-Almirall, P. ed. al, 2017), con el objetivo de detectar aquellas zonas que eran más susceptibles de recibir ayudas de carácter social. Gracias a dicho mapa, fue posible en 2017, por parte del Ayuntamiento de Barcelona, iniciar un programa de ayudas a la rehabilitación de edificios destinado a aquellas comunidades en situaciones complejas de vulnerabilidad que necesitaban rehabilitar sus edificios por motivos importantes de seguridad y habitabilidad (Ayuntamiento de Barcelona, 2018). La detección de aquellas fincas que necesitaban ser rehabilitadas se hizo mediante un amplio estudio de prediagnosis de la edificación existente (Cornadó, C. et.al., 2019; Cornadó, C. et.al., 2021; Vima-Grau, S., 2019).

El estudio que aquí se presenta se realizó en el barrio del Carmel de Barcelona, uno de los barrios que están incluidos en el Pla de Barris y en el que se encuentran zonas identificadas como vulnerables en el contexto de la ciudad de Barcelona. En la conformación del barrio del Carmel es clave su accidentada orografía, éste se sitúa en un terreno de muy difícil construcción, como todos los barrios que han crecido en las laderas empinadas de las colinas. Un barrio inicialmente configurado por las segundas residencias que sirvieron como lugar de veraneo hasta la posguerra, cuando empezaron a aparecer las casas auto construidas. La densificación máxima de Carmel se produjo con la ola migratoria de los años sesenta en la que se produjo el mayor crecimiento del barrio. Así, en el barrio se combinan edificios históricos, con crecimientos irregulares y con edificios plurifamiliares de promoción privada construidos en la segunda mitad del siglo XX.

La situación con respecto a la vivienda es heredera de un crecimiento informal en su origen y que, si bien se ha ido adaptando, mantiene un nivel de habitabilidad y de solidez que habría que adecuar a las condiciones actuales de vida y de exigencias normativas a las viviendas.

\section{OBJETIVOS}

El objetivo principal de la investigación era el de caracterizar la edificación del barrio del Carmel desde el punto de vista de sus condiciones de habitabilidad y estado de conservación creando una metodología específica para ello. Además, se plantean los siguientes objetivos secundarios:

- Realizar una evaluación del parque edificado en el barrio del Carmel a partir de la explotación de bases de datos para detectar situaciones de riesgo de infravivienda.

- Realizar una prediagnosis de la edificación para establecer niveles de prioridad en su necesidad de rehabilitación.

- Detectar y categorizar las diferentes situaciones de infravivienda que se encuentran en el ámbito de estudio. 


\section{METODOLOGÍA}

La investigación se centra en la edificación residencial del barrio del Carmel (figura 1) y se plantea como una aproximación a partir de la explotación de las bases de datos existentes y estudios previos disponibles, combinada con el estudio de campo desde el exterior de los edificios para la evaluación del riesgo de infravivienda. De esta manera se definen las siguientes fases de estudio:

- Fase 1: caracterización inicial y desarrollo de criterios e indicadores de riesgo de infravivienda

- Fase 2: campaña in situ

- Síntesis
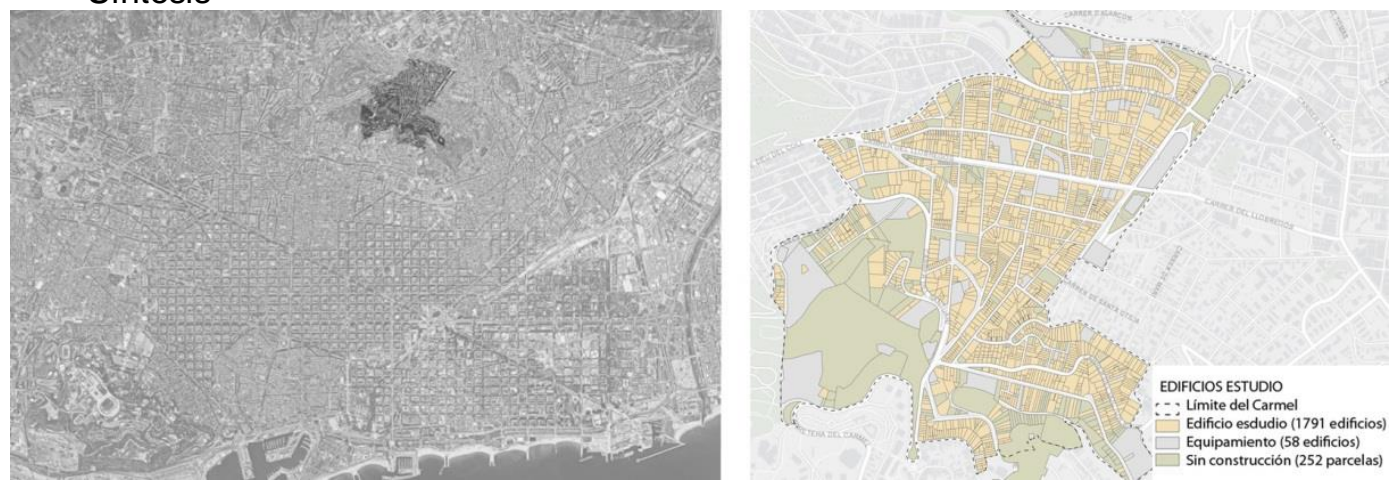

Figura 1: barrio del Carmel en el contexto de Barcelona (izq.) y edificación residencial incluida en el ámbito de estudio (der.). Fuente: elaboración propia

Fase 1: caracterización inicial y desarrollo de criterios e indicadores de riesgo de infravivienda

Esta primera fase se realiza a partir de la explotación de las diferentes bases de datos y estudios previos disponibles. A partir de éstas, se establecen y consensuan unos criterios e indicadores del riesgo de infravivienda a partir de varias reuniones con los técnicos municipales y agentes sociales del barrio y distrito. En la tabla 1, se listan los criterios e indicadores especificando las fuentes de información de cada uno de ellos.

Tabla 1: Criterios e indicadores para la obtención de un índice de riesgo de infravivienda. Fuente: elaboración propia

\begin{tabular}{|c|c|}
\hline Criterio o indicador & Fuente \\
\hline Año de construcción de la edificación & Catastro \\
\hline $\begin{array}{l}\text { Antigüedad efectiva (ver apartado } \\
\text { resultados) }\end{array}$ & Catastro \\
\hline Grado de empadronamiento & $\begin{array}{l}\text { Censo de viviendas vacías en la ciudad de Barcelona: el } \\
\text { Carmel } \\
\text { Catastro } \\
\text { Padrón }\end{array}$ \\
\hline Superficie de la vivienda & Catastro \\
\hline Superficie por habitante & $\begin{array}{l}\text { Catastro } \\
\text { Padrón }\end{array}$ \\
\hline Categoría catastral & Catastro \\
\hline Usos de los locales en planta baja & $\begin{array}{l}\text { Catastro } \\
\text { Censo de locales de planta baja }\end{array}$ \\
\hline $\begin{array}{l}\text { Viviendas bajo rasante } \\
\text { Índice de vulnerabilidad }\end{array}$ & $\begin{array}{l}\text { Catastro } \\
\text { "Estudio y detección en la ciudad de Barcelona de } \\
\text { ámbitos de vulnerabilidad residencial" (Garcia-Almirall, et. } \\
\text { al., 2017) }\end{array}$ \\
\hline
\end{tabular}

Fase 2: campaña in situ 


\section{Indicadores relacionados con la intensidad de los usos de vivienda (F1)}

Los indicadores y criterios que nos dan información sobre el uso más o menos intensivo de las viviendas son: el grado de empadronamiento, la superficie por vivienda y la superficie por habitante (Figura 3). Del estudio de los tres indicadores se concluye que es un barrio habitado, con un grado de empadronamiento elevado, con existencia de viviendas de dimensiones reducidas (inferiores a $36 \mathrm{~m} 2$ ) y medianas-pequeñas (entre $36-56 \mathrm{~m} 2$ ) abundantes, con una distribución que se hace más presente en la zona alta del barrio, correspondiente al extremo sur este del barrio. Se observan algunos casos de densidades muy elevadas, con superficies por habitante inferiores a $9 \mathrm{~m} 2$ y de 9 a $18 \mathrm{~m} 2$ por habitante.

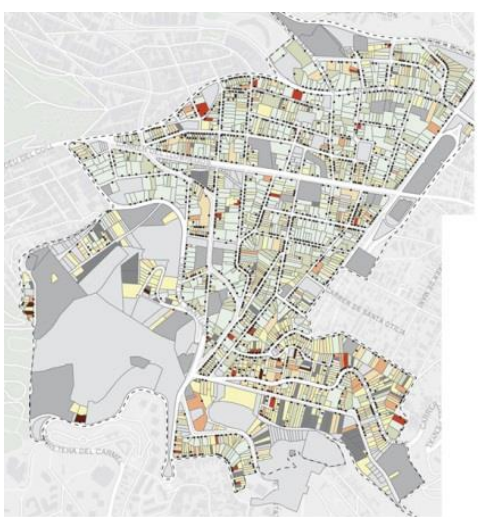

Grado de empadronamiento

\begin{tabular}{|l}
\hline \\
entre $100 \%$ y $75 \%$ \\
entre $75 \%$ y $50 \%$ \\
entre $50 \%$ y $25 \%$ \\
entre $25 \%$ y $0 \%$ \\
más empadronamiento que vivienda \\
empadronamiento sin vivienda \\
vivienda sin empadronamiento \\
sin empadronamiento ni viv. \\
equipamiento \\
sin construcción
\end{tabular}

Figura 3: Indicadores relacionados con la intensidad de los usos de vivienda. Fuente: elaboración propia

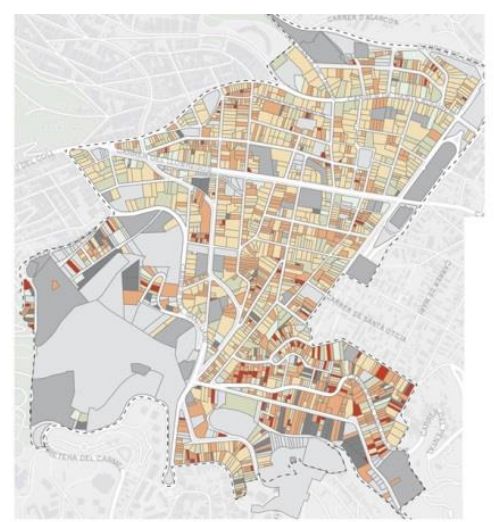

Superfície viviendas

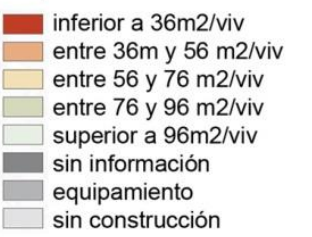

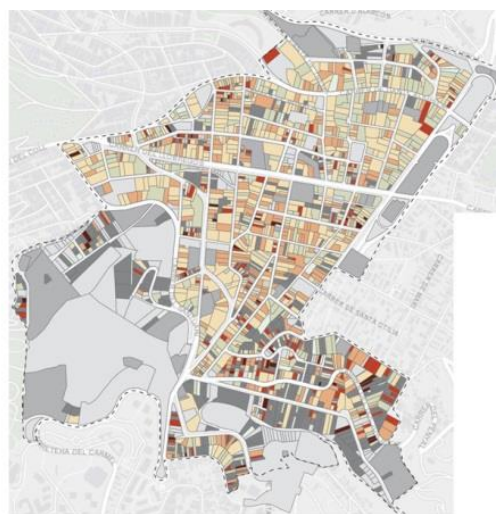

Superfície por habitante

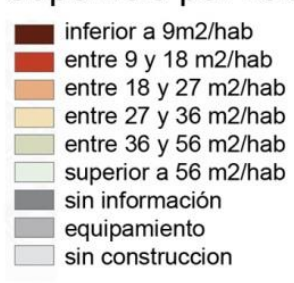

\section{Categoría catastral e índice de vulnerabilidad (F1)}

La tipología constructiva, elaborada según las Normas Técnicas de Valoración (Gobierno de España, 1993), contiene información sobre la categoría catastral, que es el factor que permite calcular el valor catastral de cada construcción en función de la su tipología. La categoría catastral es un valor entre 1 y 9 , siendo el valor 1 el que corresponde a una construcción de un mayor standard y 9 el que menos. El "Estudio y detección en la ciudad de Barcelona de ámbitos de vulnerabilidad residencial" (Garcia-Almirall, et. al., 2017) analizó la vulnerabilidad socioresidencial en cuatro ejes: socioeconómico (desahucios, ayudas para pago del alquiler, pensiones, etc.), socioespacial (movilidad, población infantil, índice de envejecimiento, etc.), urbanístico (estado del edificio, accesibilidad, actividad rehabilitadora, etc.) y sociourbanístico (tipo de propiedad, actividad urbana, etc.). Con un total de 40 indicadores se estableció un índice de vulnerabilidad para todas las parcelas donde la vivienda era el uso principal. Del estudio de estos dos indicadores se concluyó que la parte sureste del barrio y central eran las que concentraban los valores más altos de estos dos últimos indicadores.

\section{Estado de conservación de los edificios: prediagnosis de la edificación (F2)}

Se realiza un estudio exhaustivo sobre el estado de conservación de los edificios residenciales del Barrio, replicando la metodología mencionada anteriormente. Éste consistió en una prediagnosis del estado de conservación de la edificación realizada desde el exterior y estableciendo cinco índices correspondientes al nivel de prioridad de necesidad de rehabilitación de cada edificio:

\section{- 1: rehabilitación urgente}




\section{A \\ ARQUITECNO \\ Còssima Cornadó, Pilar Garcia-Almirall, Jordi Casals y Amaya Caballero}

- 1,5: rehabilitación necesaria

- 2: rehabilitación recomendable

- 2,5: realización de acciones de mantenimiento

- 3: mantenimiento habitual aconsejable

Además, se tiene en cuenta para la prediagnosis y se registran una serie de indicadores observables en los edificios (daños y deficiencias): presencia de humedades de capilaridad, presencia de humedades por filtración, elementos o revestimientos de fachada inestables, presencia de redes o elementos de protección, grietas en muros de fachada, grietas en el revestimiento, ventanas sin carpintería y / o vidrio o ventanas tapiadas y / o carpinterías en mal estado e instalaciones en mal estado.

Los resultados de la prediagnosis realizada se muestran en la figura 4. Se han podido detectar un $36 \%$ de las fincas existentes en el barrio del Carmel que presentan un grado elevado de necesidad de rehabilitación (índices 1, 1.5 y 2). Estos se encuentran generalmente distribuidos por todo el ámbito de estudio si bien se aprecia una mayor concentración en las zonas más elevadas del barrio (zona sureste). Aproximadamente un 15\% de los edificios observados desde el exterior podrían presentar situaciones de riesgo, ya sea global del edificio o parcial (índices 1 y 1.5), estos, igualmente, a pesar de estar distribuidos por todo el barrio abundan más por las zonas de cota más alta. Cabe decir que un $63 \%$ de los edificios se encuentran en un correcto estado de conservación, con lo que un mantenimiento habitual sería suficiente. Estos se concentran en las zonas de cota más baja del barrio.

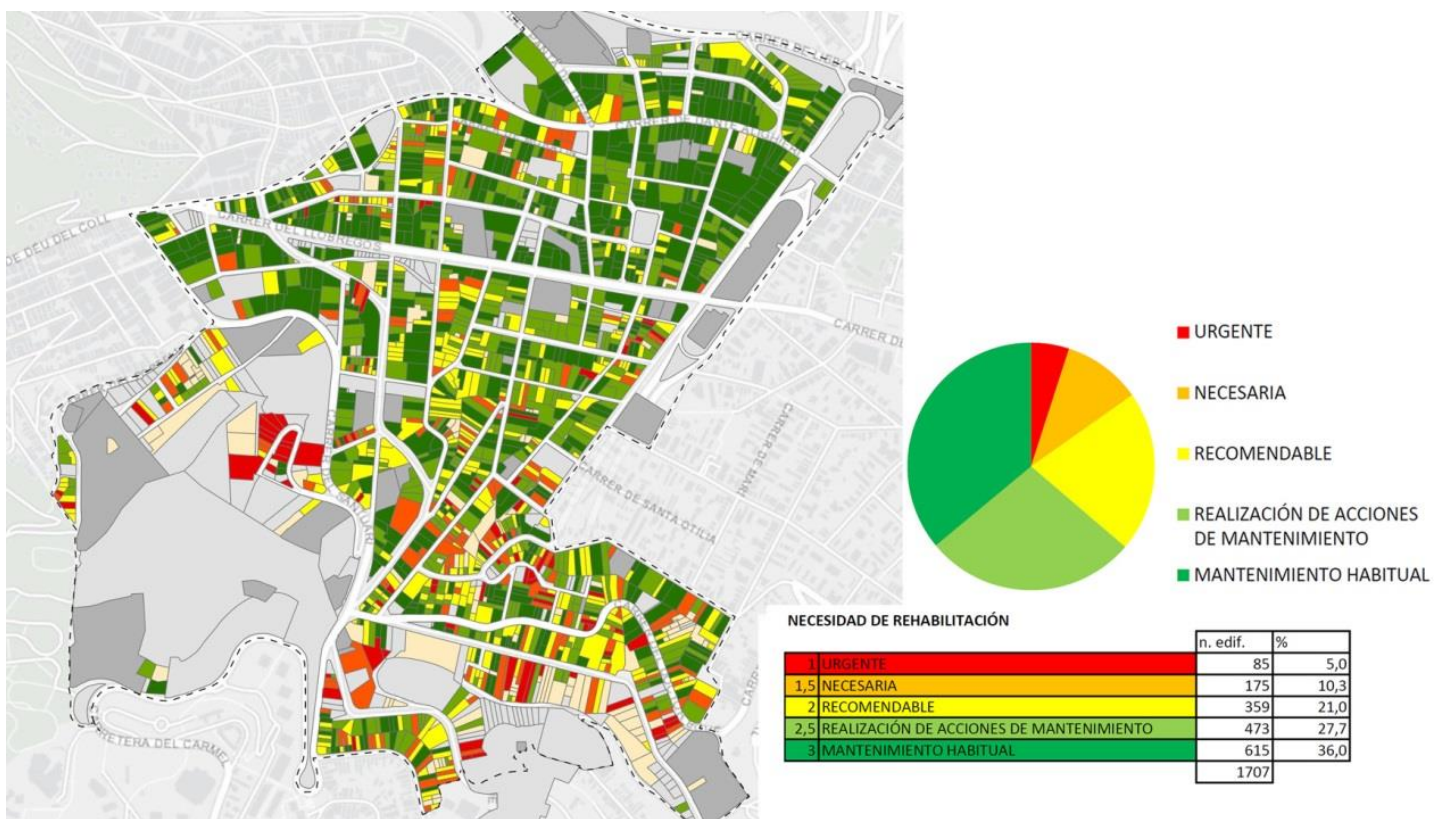

Figura 4: Índice de necesidad de rehabilitación de los edificios residenciales de el Carmel. Fuente: elaboración propia

Tabla 2: Daños y deficiencias de los edificios observables desde el exterior. Fuente: elaboración propia

\begin{tabular}{lll}
\hline Daños y deficiencias detectados & N. edif. & $\%$ \\
\hline Humedades de capilaridad & 204 & $12 \%$ \\
Humedades por filtración & 391 & $23 \%$ \\
Presencia de elementos inestables & 514 & $30 \%$ \\
Redes de protección (medidas cautelares de protección) & 34 & $2 \%$ \\
Grietas en muros & 312 & $18 \%$ \\
Grietas en revestimientos & 557 & $33 \%$ \\
Carpinterías en mal estado & 36 & $2 \%$ \\
Instalaciones en mal estado. & 11 & $1 \%$ \\
\hline
\end{tabular}

En cuanto a los daños y / o deficiencias de los edificios que han podido ser detectados desde el exterior (Tabla 2) abundan la presencia de elementos inestables (presentes en un $30 \%$ de los 
edificios), estos son a menudo, revestimientos agrietados que presentan situaciones de inestabilidad, recubrimientos de ladrillo en situación de desprenderse y cornisas y balcones en mal estado. Aunque se han detectado estas situaciones, únicamente un $2 \%$ de los edificios observados disponen de redes o elementos de protección cautelares.

\section{Deficiencias de accesibilidad (F2)}

Se han tenido en cuenta aquellas carencias de accesibilidad que tienen que ver con el acceso al edificio y su entorno más inmediato (Figura 5). Estas son:

- no consecución de los mínimos de accesibilidad desde el exterior establecidos en la normativa española (Gobierno de España, 2010) generalmente por la presencia de escalones en la entrada al edificio. Esta deficiencia ha sido observada en un total de 1432 edificios.

- acceso al edificio a través de una calle o pasaje público o privado de anchura reducida, observado en un total de 149 edificios.

- acceso al edificio a través de una calle o pasaje público o privado que tiene como única alternativa el uso de escaleras, observado en un total de 126 edificios.

En cuanto al acceso del edificio, es muy habitual encontrar uno o más escalones en la entrada (77\% de los edificios residenciales del barrio), por tanto no se alcanzan los mínimos de accesibilidad exigibles hoy en día. Sin embargo, este hecho se podría solucionar en muchos casos con la sustitución del escalón de la entrada por una rampa en el vestíbulo. La accidentada orografía del Carmel es muy patente en algunas calles donde la pendiente es muy acusada o, incluso, todo el ancho de la calle está ocupado por escaleras. En estos casos la deficiencia de accesibilidad no se puede solucionar interviniendo en el edificio. Otra característica de la parcelación del barrio del Carmel, derivada de la orografía, es el elevado número de parcelas "interiores". Los edificios de estas parcelas no tienen el acceso desde la calle sino que a ellos se accede a través de pasajes, pasos, escaleras, de diversa naturaleza. Estos casos suelen implicar deficiencias en la accesibilidad con anchuras insuficientes y escaleras. En algunos casos, los pasos a las parcelas interiores son de libre acceso público, en otros, éstos están cerrados sólo a los vecinos, lo que ha imposibilitado la visita.

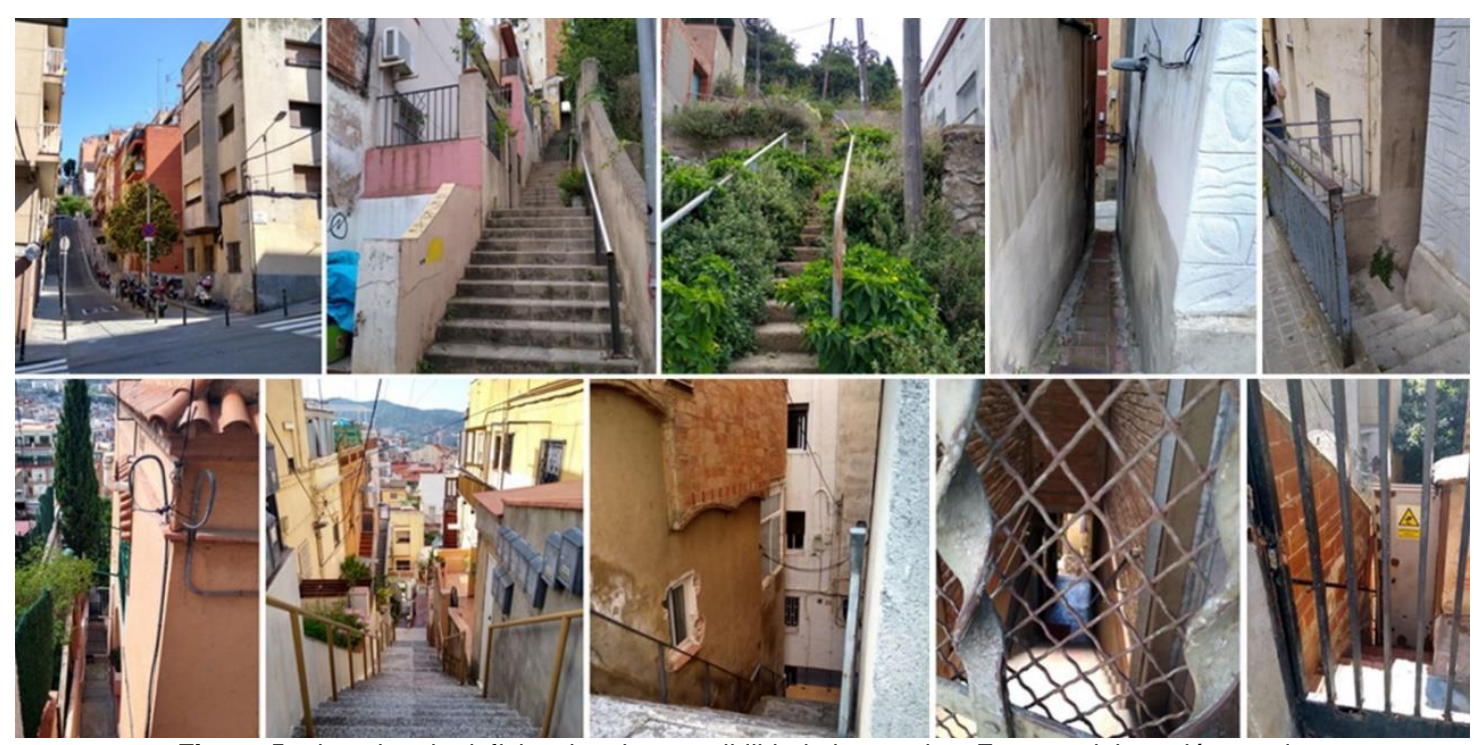

Figura 5: ejemplos de deficiencias de accesibilidad observadas. Fuente: elaboración propia

\section{Indicios de deficiencias en la ventilación e iluminación (F2)}

Las diferencias de cota entre varios puntos de una misma parcela pueden hacer que pisos semisótano y sótano, incluso de varias plantas, se encuentren sobre rasante en parte de la planta del edificio, pudiendo ventilar e iluminar por esa zona. La orografía también puede hacer que determinados puntos de la vivienda que se encuentren bajo rasante busquen una ventilación e iluminación en situaciones extremas encontrándose numerosos edificios (192 edificios) con ventanas a ras de acera. Contrariamente, cabe la sospecha de la existencia de cámaras 0 
habitaciones que, encontrándose bajo rasante, carecen de ventilación, hecho no detectable con un tipo de campaña realizada únicamente desde el exterior.

Otra situación de ventilación deficiente de las piezas de la vivienda que quedan bajo rasante es la ventilación e iluminación mediante un patio delantero adyacente a fachada. Estos patios suelen tener una anchura reducida y una única planta de profundidad, aunque en algunas ocasiones el patio es más ancho y de dos o tres plantas de profundidad.

Además de estas situaciones (Figura 6) también se han registrado aquellos casos donde el número de ventanas en fachada se ha observado aparentemente insuficiente para garantizar las condiciones de iluminación y ventilación de las viviendas.

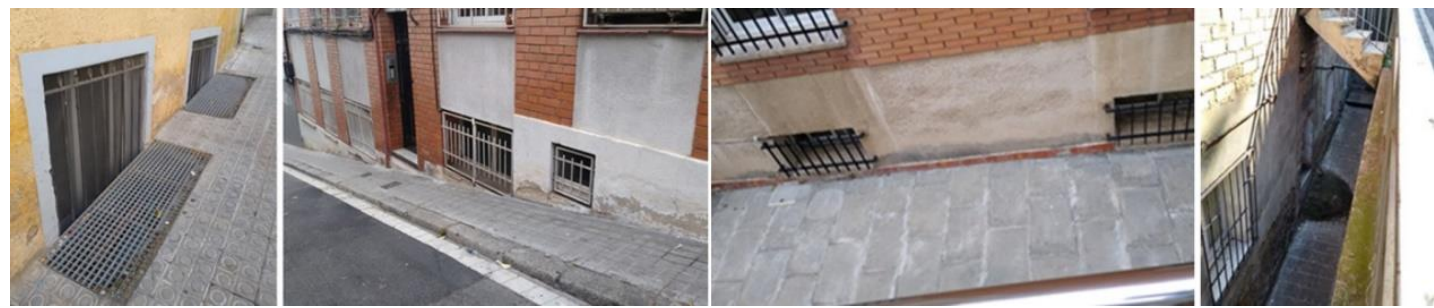

Figura 6: ejemplos de deficiencias de ventilación e iluminación observadas. Fuente: elaboración propia

\subsection{Usos en planta baja (F2)}

Se han registrado los diferentes usos de los edificios en planta baja, distinguiendo entre:

- Uso con acceso público: incluye comercios, servicios y equipamientos públicos, detectado en 308 edificios

- Uso sin acceso público: mayoritariamente vivienda, aunque también hay otros usos como taller, oficinas, almacén o garaje, detectado en 1234 edificios

- Sin uso: locales vacíos, detectado en 162 edificios

Existe una gran parte del barrio que tiene viviendas en planta baja (1.086 edificios), lo que considerando también la accidentada orografía puede dificultar las condiciones de ventilación. La actividad comercial se concentra en la parte baja del barrio. De aquellos usos sin acceso público: vivienda y otros (talleres, oficinas, almacenes, etc.) que también podrían ser susceptibles de alojar un uso irregular de vivienda se ha hecho una distinción en función de su superficie.

\section{8 Índice conjunto}

Para la obtención de un índice conjunto síntesis que indique el riesgo de existencia de infravivienda se han ponderado los indicadores de las dos fases, tal como se indica en la tabla 3 , tras consensuarse con los técnicos y agentes de distrito. Los resultados muestran una concentración mayor de posible existencia de infravivienda en las zonas más altas del barrio (Figura 7), correspondientes a la zona sur este. También una incidencia más alta en parcelas interiores o difícilmente accesibles a través de pasajes privados.

Tabla 3: Indicadores y ponderación para la elaboración del índice conjunto de riesgo de infravivienda. Fuente: elaboración propia

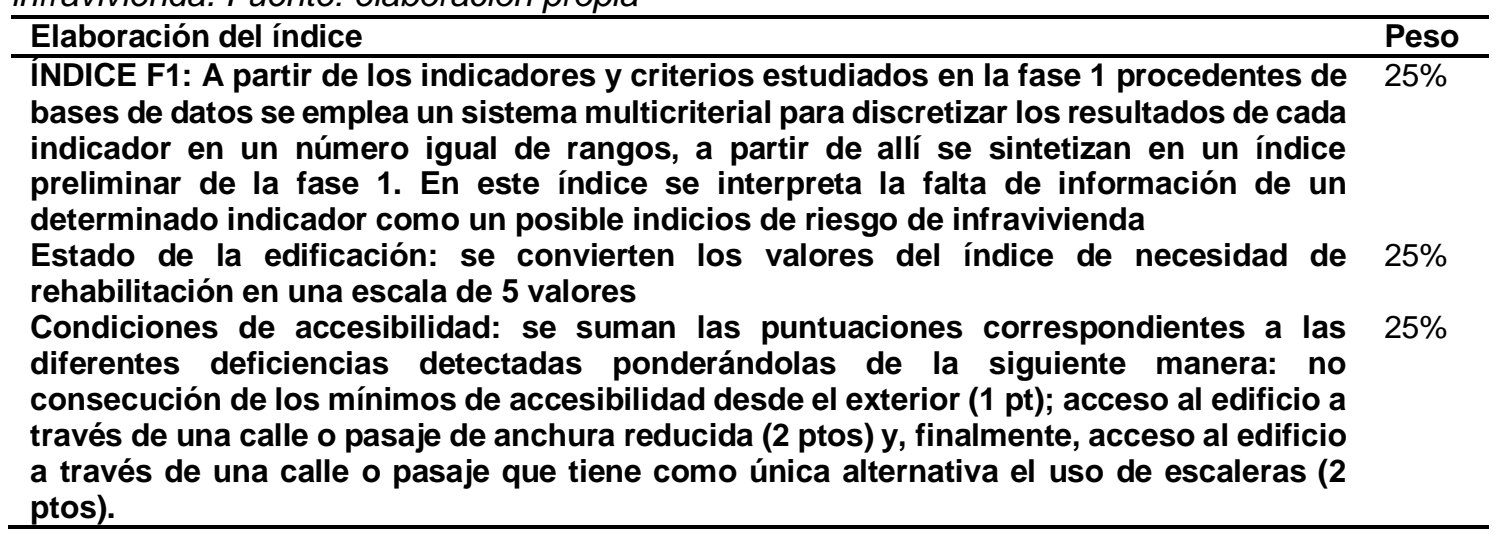


Condiciones de iluminación y ventilación: se establece la siguiente puntuación: los edificios que ventilan a través de ventanas de sótano que dan directamente a la calle se les ha asignado 5 puntos, a la ventilación de plantas sótano a través de patios frontales adyacentes se les ha asignado 4 puntos, y aquellos edificios que presentan aparente una cantidad de ventanas insuficientes les ha asignado una puntuación de 3.

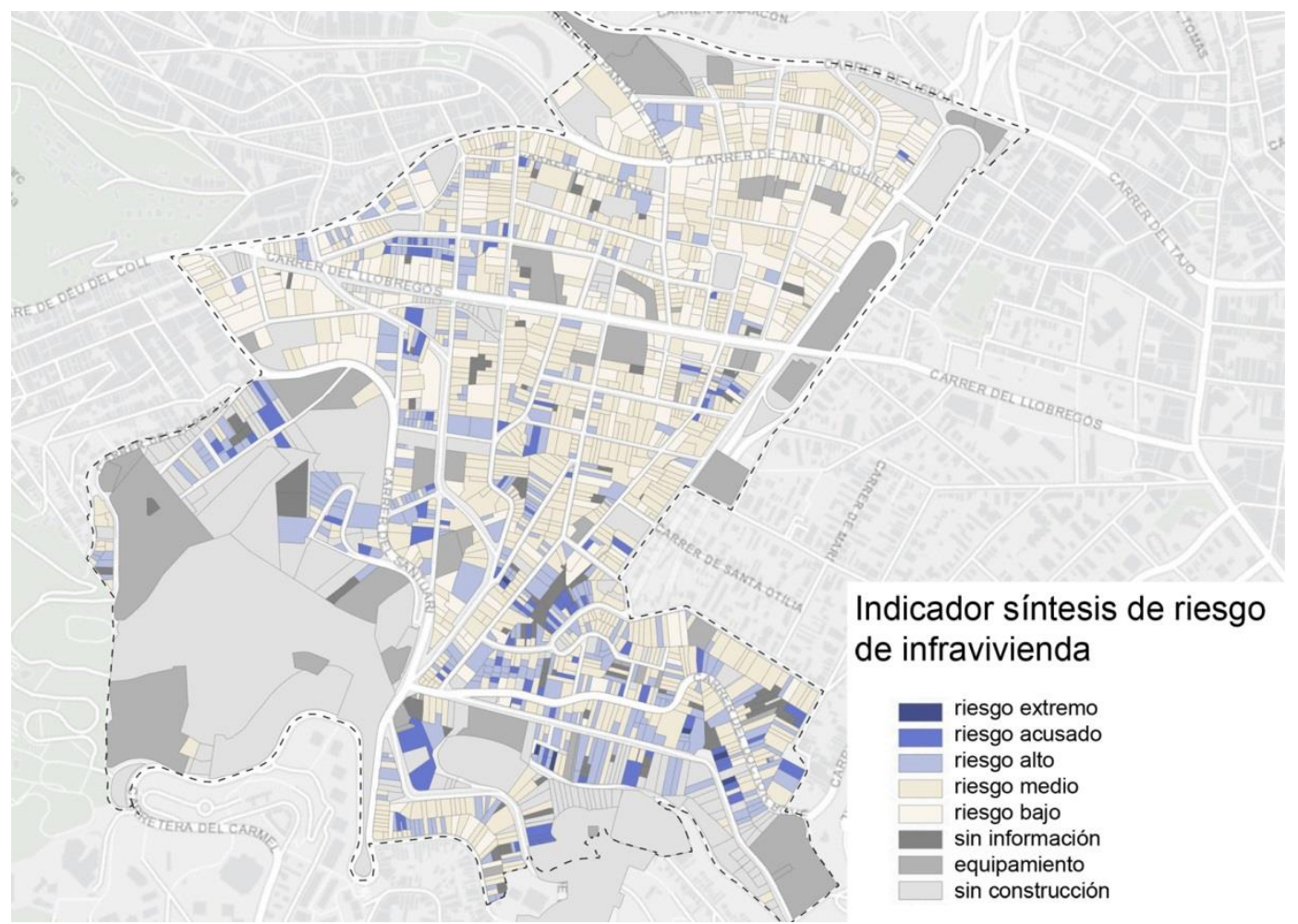

Figura 7: Indicador síntesis de riesgo de infravivienda. Fuente: elaboración propia

\section{CONCLUSIONES}

El concepto de infravivienda indica unas características de la edificación destinada a vivienda por debajo de los requerimientos normativos de seguridad, accesibilidad universal y habitabilidad. Por lo tanto, además del estado de conservación de la edificación y la presencia de determinados tipos de daños en la edificación, como por ejemplo, la presencia de humedades o instalaciones deficientes, que pueden indicar deficiencias de seguridad y salubridad, se deben considerar otros aspectos que inciden en las condiciones de habitabilidad. Algunos de estos aspectos son difícilmente detectables desde el exterior como, por ejemplo, todos aquellos que tienen que ver con las dimensiones de las diferentes piezas que conforman la vivienda y su distribución. En cambio, sí se pueden detectar otros.

El uso de una metodología híbrida cuantitativa y cualitativa, que combina la explotación de datos procedentes de diferentes bases de datos con la inspección in situ desde el exterior de los edificios permite profundizar en el conocimiento de los mismos y detectar aquellas situaciones anómalas que pueden llevar a condiciones de infravivienda.

En el caso del barrio del Carmel, se constata como gran parte de los originantes de dicho riesgo de infravivienda se encuentran en la conformación urbana del barrio (orografía, parcelaciones interiores, etc.) que son difícilmente solventables si se quiere mantener un uso de vivienda en condiciones mediante la intervención en los edificios existentes, por lo que se sugiere abordar cambios de mayor calado.

La investigación ha permitido disponer de una cartografía indicativa del riesgo de infravivienda en el barrio del Carmel en Barcelona, de utilidad para futuros programas de incentivos para la 
rehabilitación de edificios. Además, proporciona una metodología válida replicable en otras situaciones y ubicaciones.

Agradecimientos: Esta investigación se enmarca en el proyecto: Re-Inhabit. Indicadores socioespaciales para la mejora del stock habitacional en zonas vulnerables. Criterios de actuación en los casos de las áreas metropolitanas de Barcelona y Bilbao. RTI 2018-101342-B-I00, financiado por el min. de Ciencia, Innovación y Universidades (Gob. de España)

\section{REFERENCIAS}

Alcalá, L., Páez, M. \& Andújar-Llosa, A. (2013). Vulnerabilidad Social y residencial en la Cañada Real del Municipio de Madrid. XI Congreso Español de Sociología. Crisis y cambio: propuestas desde la Sociología.

Aragonés, J. \& Rodríguez, J. (1992). Satisfacción residencial en ámbitos de infravivienda. Psicothema, 4. 329-341.

Ayuntamiento de Barcelona (2015). Plan de Barrios. https://pladebarris.barcelona/es/inicio

Ayuntamiento de Barcelona (2018) Ayudas a la rehabilitación para fincas de alta complejidad. . https://habitatge.barcelona/es/servicios-ayudas/rehabilitacion/ayudas/fincas-altacomplejidad

Cornadó, C., Garcia-Almirall, P., Vima-Grau, S., Vila, G. \& Uzqueda, A. (2017) Methodology for the Detection of Residential Vulnerable Areas - the Case of Barcelona. IOP Conf. Series. Materials Science and Engineering, 245. doi:https://doi.org/10.1088/1757899X/245/4/042062

Cornadó, C., Vima-Grau, S., Martín, E., Garcia-Almirall, P \& Uzqueda, A. (2019) Methodology for the Pre-diagnosis of Residential Buildings in Vulnerable Areas in the City of Barcelona. IOP Conf. Series: Materials Science and Engineering, 471. doi:https://doi.org/10.1088/1757899X/471/7/072030. 2019

Cornadó C., Vima-Grau S. \& Garcia-Almirall P. (2021) Pre-diagnosis Protocol for Large Residential Building Stock. The Case of Barcelona's Vulnerable Areas. In: Delgado J. (eds) Hygrothermal Behaviour and Building Pathologies. Building Pathology and Rehabilitation, vol 14. Springer, Cham. https://doi.org/10.1007/978-3-030-50998-9_6

Garcia-Almirall, P., Vila, G., Vima-Grau, S. \& Uzqueda, A. (2017). Estudi i detecció a la ciutat de Barcelona d'àmbits de vulnerabilitat residencial. Ajuntament de Barcelona

Gobierno de España (1993) Real Decreto 1020/1993, de 25 de junio, por el que se aprueban las normas técnicas de valoración y el cuadro marco de valores del suelo y de las construcciones para determinar el valor catastral de los bienes inmuebles de naturaleza urbana

Gobierno de España (2010) Ley 38/1999 de 5 de noviembre, de Ordenación de la Edificación, de la que nace el Código Técnico de la Edificación. Documento Básico, Seguridad de Utilización y Accesibilidad (CTE-DB-SUA), de febrero de 2010, pp. 1-46.

Gobierno de España (2015) Real Decreto Legislativo 7/2015, de 30 de octubre, por el que se aprueba el texto refundido de la Ley de Suelo y Rehabilitación Urbana. «BOE» núm. 261, de 31 de octubre de 2015, pp 103232-103290 https://www.boe.es/eli/es/rdlg/2015/10/30/7

Vima-Grau, S., Cornadó, C., \& Garcia-Almirall, P. (2019). Socio-spatial analysis of the vulnerable urban fabric in the city of Barcelona. VITRUVIO - International Journal of Architectural Technology and Sustainability, 4(2), 75-89. doi:https://doi.org/10.4995/vitruvioijats.2019.12559 